\title{
Delayed Onset
}

National Cancer Institute

\section{Source}

National Cancer Institute. Delayed Onset. NCI Thesaurus. Code C161521.

The start of an event or episode that is characterized as occurring later or at a relatively long period of time after a reference time. 